Philipp Krug, Dominika Langenmayr, Niklas Rauhut, Finn Ole Schmude, Thea Seitz, Douglas Strasoldo, Moritz Withoeft

\title{
Steuerehrlichkeit und Steuerlotterien
}

\begin{abstract}
Für die Einnahmenseite der Staatsfinanzen ist nicht die aggregierte rechnerische Steuerschuld ausschlaggebend, sondern das tatsächliche Steueraufkommen. Diese beiden Größen fallen durch die „Steuerlücke“ auseinander, die das Ausmaß an Steuerhinterziehung beschreibt. Insbesondere bei der Umsatzsteuer reduziert mangelnde Steuerehrlichkeit das Steueraufkommen vieler Staaten. Während in Deutschland z. B. die Registrierkassenpflicht und die Kassenbonpflicht als Maßnahmen eingeführt wurden, setzen andere Staaten auch auf Steuerlotterien. Solche Lotterien werden diskutiert und konkrete Ausgestaltungsvorschläge gemacht, die mithilfe moderner Technologien auch einen positiven Effekt auf die Steuermoral haben können. Das zugrundeliegende Konzept wurde von Schüler:innen der Deutschen Berufsschule Hong Kong für den Schülerwettbewerb YES! Young Economic Summit entwickelt.
\end{abstract}

Die Europäische Kommission schätzt in ihrer neuesten Studie, dass die Mehrwertsteuerlücke in der EU 2018 bei 140 Mrd. Euro liegt. Nachdem diese Zahl in den Vorjahren kontinuierlich gesunken war, wird für das Corona-Jahr ein deutlicher Anstieg erwartet, was vor allem an der Ausweitung des informellen Sektors liegen dürfte. Die Steuerlücke ist unter den Mitgliedstaaten der EU sehr heterogen und reicht von nur 0,7\% der rechnerischen Steuerschuld in Schweden bis hin zu 33,8\% in Rumänien (vgl. Abbildung 1). Der Median liegt bei 9,2\%. In Deutschland beläuft sich der Wert auf 8,6\%, was 2018 einem absoluten Fehlbetrag von etwa 22 Mrd. Euro entspricht (Europäische Kommission, 2020). Das Ausmaß von Umsatzsteuerhinterziehung und der damit verbundene Aufkommensausfall gebietet eine detaillierte Auseinandersetzung mit potenziellen Gegenmaßnahmen. Die Grundlage dafür ist ein ausgeprägtes Verständnis der Determinanten von Steuerehrlichkeit.

\section{Steuerehrlichkeit und ihre Determinanten}

In einer wegweisenden Studie aus dem Jahr 1972 modellieren Michael G. Allingham und Agnar Sandmo Steuerhinterziehung als Entscheidung unter Unsicherheit. Die Steuerschuldner:innen stehen vor der Entscheidung, sich gesetzeskonform zu verhalten und somit die Steuerpflicht vollumfänglich zu erfüllen oder aber Steuern zu hinterziehen, was das Risiko mit sich bringt, entdeckt und bestraft zu werden. Neben dem Steuersatz, der beeinflusst, wie

(C) Der/die Autor:in 2021. Open Access: Dieser Artikel wird unter der Creative Commons Namensnennung 4.0 International Lizenz veröffentlicht (creativecommons.org/licenses/by/4.0/deed.de).

Open Access wird durch die ZBW - Leibniz-Informationszentrum Wirtschaft gefördert. viel durch Steuerhinterziehung gespart werden kann, sind die Instrumente des Staates hier also die Höhe der Strafe sowie die Anstrengungen, Steuerhinterziehung überhaupt aufzudecken, was wiederum die Entdeckungswahrscheinlichkeit beeinflusst (Allingham und Sandmo, 1972).

Die Literatur diskutiert mit der Steuermoral auch einen weiteren Einflussfaktor auf Steuerehrlichkeit. Unter Steuermoral versteht man grundsätzlich nicht finanzielle Aspekte, die die Einstellung von Individuen gegenüber Steuerhinterziehung beeinflussen. In einer für die deutsche Bevölkerung repräsentativen Umfrage geben z. B. $11 \%$ an, dass sie Steuerhinterziehung in Ordnung finden (Doerrenberg und Peichl, 2020). Der Steuermoral zugrunde liegende Mechanismen können in fünf verschiedene Typen unterteilt werden, die für unterschiedliche Indivi-

Philipp Krug ist wissenschaftlicher Mitarbeiter an der Katholischen Universität Eichstätt-Ingolstadt und dem KU Research Institute for Taxation.

Prof. Dr. Dominika Langenmayr lehrt dort sowie an der WU Wien und ist CESifo Research Fellow.

Niklas Rauhut, Finn Ole Schmude, Thea Seitz, Douglas Strasoldo und Moritz Withoeft sind Schüler:innen der Deutschen Berufsschule Hong Kong. 
duen von sehr heterogener Bedeutung sein können (Luttmer und Singhal, 2014).

Die Steuermoral kann erstens auf der intrinsischen Motivation, sich an Gesetze zu halten, basieren. Dem liegt zugrunde, dass ehrliches Verhalten ein positives Selbstbild oder das Empfinden von Stolz auslösen kann. Ein weiterer Kanal ist zweitens Reziprozität. Empfindet man Steuerzahlungen als Teil eines Art Vertrages zwischen sich selbst und dem Staat, für die man im Gegenzug - wenn auch ohne eine unmittelbare Verbindung - Leistungen des Staates erhält, so erhöht dies die Steuermoral (Doerrenberg und Peichl, 2020). Drittens kann die Steuerehrlichkeit eines Individuums durch Peer-Effekte und soziale Normen direkt vom Verhalten anderer abhängig sein. Allerdings kann das Bedürfnis, dem Verhalten anderer zu folgen, die Steuermoral nicht nur positiv, sondern auch negativ beeinflussen, wenn sich die relevante Peergroup durch eine besonders schlechte Steuerehrlichkeit auszeichnet (z.B. Paetzold und Winner, 2016). Grundlegende soziale Normen, die über einen langen Zeitraum in einer Gesellschaft bestehen, verkörpern viertens kulturelle Determinanten der Steuermoral. Zuletzt können auch fünftens imperfekte Informationen und Fehleinschätzungen, z. B. in Bezug auf die Häufigkeit von Steuerprüfungen, Abweichungen von dem Verhalten erklären, das der Allingham-Sandmo-Rahmen vorhersagt (Allingham und Sandmo, 1972).

Diese Typologie bietet nicht nur Ansatzpunkte, Heterogenität in der Steuermoral zwischen Individuen, sondern auch zwischen Regionen im internationalen Vergleich zu erklären. Basierend auf Daten des World Value Survey stellt die OECD eine ausgeprägte Steuermoral für OECDStaaten und Lateinamerika fest, wohingegen Afrika und Osteuropa deutlich schlechter abschneiden (OECD, 2019).

\section{Steuerlotterien: Theorie und Praxis}

Steuerlotterien wurden in einer Vielzahl von Ländern bereits als Instrumente gegen Mehrwertsteuerhinterziehung eingesetzt. Während Taiwan bereits 1951 eine Kassenbonlotterie einführte, gingen in der jüngeren Vergangenheit auch mehrere EU-Länder diesen Weg. Im vergangenen Jahrzehnt führten Griechenland, Italien, Kroatien, Lettland, Litauen, Polen, Portugal, Rumänien, Slowenien, die Slowakei und Tschechien Lotterien ein. Dabei fällt auf, dass vor allem Länder mit hohen Steuerlücken aktiv wurden (vgl. Abbildung 1).

Die Grundidee der Kassenbonlotterie ist, den Konsument:innen einen Anreiz zu bieten, den Kassenbon zu verlangen. Der Kassenbon (oder die zugrunde liegende Transaktion) berechtigt zur Teilnahme an einer Lotterie, fungiert dabei als Los. In der Konsequenz wird vermieden, dass Transaktionen bei Barzahlung nicht registriert wer-

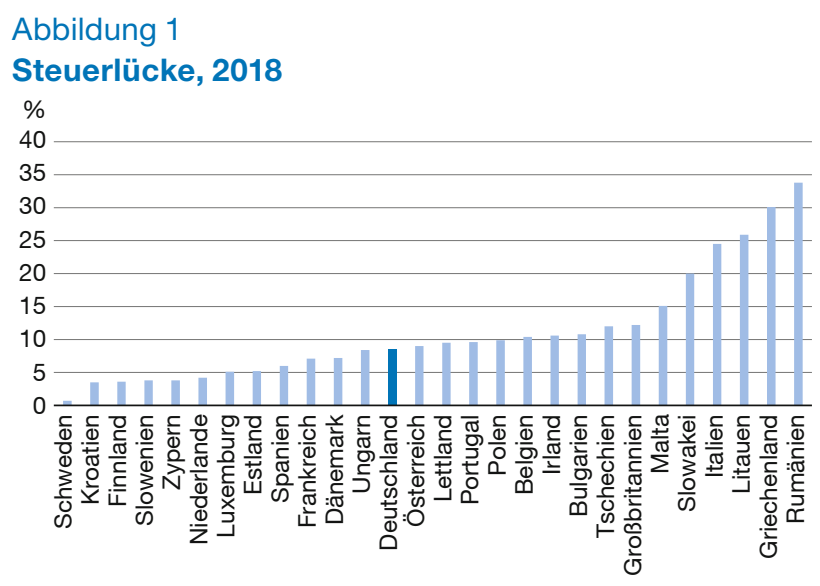

Quelle: Europäische Kommission (2020).

den und daher keine Umsatzsteuer abgeführt wird. Zwar ist der Bargeldeinsatz seit Beginn der Corona-Pandemie zurückgegangen, 2020 wurden in Deutschland aber noch immer 60 \% der Käufe im stationären Handel mit Bargeld getätigt (Deutsche Bundesbank, 2021).

Der Reduzierung der Steuerlücke stehen die Kosten der Lotterie gegenüber. Neben den Aufwendungen für die operative Durchführung, die durch die Skalierbarkeit digitaler Plattformen verringert werden können, müssen Lotteriepreise in ausreichender Menge und Höhe ausgelobt werden. Hierbei ist aber zu beachten, dass selbst wenn sich die ökonomischen Anreize für die Konsument:innen nicht in bedeutendem Maße verändern, d.h. wenn der Erwartungswert eines Gewinns nahe Null ist, die Gewinnmöglichkeit dennoch im Sinne eines „Nudges“ ausreichend für eine Verhaltensänderung beim Einfordern des Kassenbons sein kann.

Eine der wenigen detaillierten, wissenschaftlichen Evaluationen von Steuerlotterien wurde im Rahmen des „Nota Fiscal Paulista"-Programms im brasilianischen Bundesstaat São Paulo durchgeführt. Bei dieser Lotterie werden Transaktionen mit der Sozialversicherungsnummer verknüpft. Je 50 US-\$ Transaktionsvolumen erhält man ein Lotterieticket. Es wird geschätzt, dass dieses Programm die gemeldete Steuerschuld um 25,9\% steigern konnte. Nach Abzug der ausgezahlten Lotteriegewinne bedeutet dies eine Steigerung des Aufkommens um 9,3\% (Naritomi, 2019).

\section{Steuerlotterien: ein Dualer Ansatz}

Im Rahmen des Schulwettbewerbs „YES! Young Economic Summit" hat sich das Team der Berufsschule Hong Kong die Weiterentwicklung von Steuerlotterien zur Aufgabe gemacht. In einem dualen Ansatz sollen nicht nur die klassischen Anreizmechanismen der Steuerlotterie zum Tragen kommen, sondern die für die Durchführung der Lotterie benötigten Plattformen weitergehend genutzt 
werden. Die Teilnahme an den Verlosungen soll durch das Scannen eines personalisierten Codes auf dem Beleg in einer Smartphone-App erfolgen. Die Verwendung der App bietet einen direkten Kommunikationskanal zu den Konsument:innen, der andernfalls z. B. durch aufwendige öffentliche Kampagnen erzeugt werden müsste.

Die App kann z.B. über die Verwendung der Steuern informieren. Auf diese Weise wird nicht nur ein Bewusstsein für die gesellschaftliche Bedeutung von Steuerzahlungen geschaffen, sondern es wird idealerweise reziprokes Verhalten hervorgerufen, das sich langfristig auch abseits der Umsatzsteuer in einer höheren Steuermoral niederschlägt. So könnte die App z.B. darüber informieren, dass mithilfe der Mehrwertsteuer Lehrkräfte, Schulbücher oder Polizeiarbeit finanziert werden. Denkbar ist, die auf dem Kassenbon ausgewiesene Steuer fiktiv in die Zahl von Schulbüchern oder Lehrkraftstunden umzurechnen, die damit finanziert werden könnten. Damit wird eine möglichst direkte Verbindung zwischen Steuerzahlungen und wichtigen Leistungen des Staats hergestellt. Die Informationen können auch variiert werden, um andere Mechanismen wie etwa positive PeerEffekte zur Steigerung der Steuermoral zu nutzen. Auch spielerische Elemente, wie die Integration eines Steuerquiz in die App sind möglich. Zudem könnte - soweit es der Datenschutz zulässt - die App teilweise individualisiert werden.

Um die Akzeptanz der Steuerlotterie durch die Unternehmen zu erhöhen, könnte man statt einer gesetzlichen Verpflichtung zur Teilnahme wie in anderen Ländern auch hier auf die Anreizwirkung einer Lotterie setzen. Teilnehmende Firmen haben dann ebenfalls die Möglichkeit, im Rahmen von Verlosungen Preise zu gewinnen. In diesem Fall erweitert sich das Anwendungsfeld der App, und die Informationsmaßnahmen können explizit auf die Schuldner:innen der Umsatzsteuer ausgerichtet werden.

Es wird deutlich, dass neben verhaltensökonomischen Aspekten auch weitergehende Überlegungen zu Datenschutz, zu rechtlichen Rahmenbedingungen, aber auch zum Umweltschutz angestellt werden müssen. Obwohl man ursprünglich von einer „Kassenbonpflicht" spricht, erlaubt der Einsatz der App auch eine Lotterie ohne physischen Beleg. Das Scannen der QR-Codes kann problemlos an einem Kassenbildschirm erfolgen - ohne Belegdruck.
Durch den aufgezeigten dualen Ansatz werden die unmittelbaren Anreizmechanismen von Steuerlotterien mit weiteren Maßnahmen zur Steigerung der allgemeinen Steuermoral kombiniert, um Effekte zu erzielen, die sowohl über die Dauer der Lotterie hinaus persistent sind als auch die Steuerehrlichkeit bei anderen Steuerarten positiv beeinflussen. Nicht zuletzt wegen der sehr effizienten Ausgestaltung in Form einer App ist dies ein vielversprechender Weg.

\section{Fazit}

Wie auch bei anderen Politikmaßnahmen, sollte die Wirkung von Steuerlotterien systematisch evaluiert werden. Nur so lassen sich zukünftig weitere Verbesserungen in der Ausgestaltung erzielen. Bisher ist die wissenschaftliche Literatur zu Steuerlotterien noch sehr übersichtlich, was sich durch die Einführung der europäischen Lotterien in den kommenden Jahren ändern könnte, wenn entsprechende Daten zugänglich gemacht werden.

Eine Chance für die Weiterentwicklung bieten digitale Technologien, mit deren Hilfe die gewonnenen Kommunikationswege mit Konsument:innen und Steuerschuldner:innen optimal genutzt werden können. Durch ein geeignetes Design der Kommunikationsinhalte können Steuerlotterien so womöglich über die unmittelbare Anreizwirkung hinaus mittel- und langfristig die Steuermoral positiv beeinflussen.

\section{Literatur}

Allingham, M. G. und A. Sandmo (1972), Income Tax Evasion - A Theoretical Analysis, Journal of Public Economics, 1(3-4), 323-338.

Deutsche Bundesbank (2021), Zahlungsverhalten in Deutschland 2020 Bezahlen im Jahr der Corona-Pandemie, https://www.bundesbank. $\mathrm{de} / \mathrm{resource/blob/855642/cabf0971e0f0697d688dcc57c0cb65d3/mL/}$ zahlungsverhalten-in-deutschland-2020-data.pdf (17. November 2021).

Doerrenberg, P. und A. Peichl (2020), Tax Morale and the Role of Socia Norms and Reciprocity - Evidence from a Randomized Survey Experiment, Working Paper.

Europäische Kommission (2020), Study and Reports on the VAT Gap in the EU-28 Member States, 18, 20.

Luttmer, E. F. P. und M. Singhal (2014), Tax Morale, Journal of Economic Perspectives, 28(4), 149-168.

Naritomi, J. (2019), Consumers as Tax Auditors, American Economic Review, 109(9), 3031-3072.

OECD (2019), Tax Morale - What Drives People and Businesses to Pay Tax?, OECD Publishing.

Paetzold, J. und H. Winner (2016), Taking the High Road? Compliance with Commuter Tax Allowances and the Role of Evasion Spillovers, Journal of Public Economics, 143, 1-14.

Title: Tax Compliance and Receipt Lotteries

Abstract: The tax gap - the difference between taxes owed and taxes paid - measures the extent of tax evasion. Lacking tax compliance reduces tax revenue in many countries, especially from value-added taxes. As a countermeasure, Germany recently introduced mandatory cash registers and obligatory receipts. At the same time, other countries use receipt lotteries. This article discusses how such lotteries can be designed to generate a positive effect on tax morale with the help of modern technologies. The underlying concept was developed by students from the German Business Faculty in Hong Kong for the Young Economic Summit (YES!) school competition.

JEL Classification: H25, H26 